\title{
Comparison of four outdoor mosquito trapping methods as potential replacements for human landing catches in western Kenya
}

\author{
Bernard Abong'o ${ }^{1,2^{*}} \mathbb{D}$, John E. Gimnig 3 , Bradley Longman², Tobias Odongo², Celestine Wekesa², \\ Amos Webwile ${ }^{2}$, Benjamin Oloo ${ }^{2}$, Mercy Nduta ${ }^{2}$, Margaret Muchoki ${ }^{2}$, Diana Omoke ${ }^{1}$, Daniel Wacira ${ }^{6}$, \\ Kevin Opondo ${ }^{2}$, Eric Ochomo ${ }^{1}$, Stephen Munga', Martin J. Donnelly ${ }^{4}$ and Richard M. Oxborough ${ }^{5}$
}

\begin{abstract}
Introduction: Longitudinal monitoring of outdoor-biting malaria vector populations is becoming increasingly important in understanding the dynamics of residual malaria transmission. However, the human landing catch (HLC), the gold standard for measuring human biting rates indoors and outdoors, is costly and raises ethical concerns related to increased risk of infectious bites among collectors. Consequently, routine data on outdoor-feeding mosquito populations are usually limited because of the lack of a scalable tool with similar sensitivity to outdoor HLC.

Methodology: The Anopheles trapping sensitivity of four baited proxy outdoor trapping methods-Furvela tent trap (FTT), host decoy trap (HDT), mosquito electrocuting traps (MET) and outdoor CDC light traps (OLT) —was assessed relative to HLC in a $5 \times 5$ replicated Latin square conducted over 25 nights in two villages of western Kenya. Indoor CDC light trap (ILT) was run in one house in each of the compounds with outdoor traps, while additional non-Latin square indoor and outdoor HLC collections were performed in one of the study villages.

Results: The MET, FTT, HDT and OLT sampled approximately 4.67, 7.58, 5.69 and 1.98 times more An. arabiensis compared to HLC, respectively, in Kakola Ombaka. Only FTT was more sensitive relative to HLC in sampling An. funestus in Kakola Ombaka (RR $=5.59,95 \% \mathrm{Cl} 2.49-12.55, P<0.001)$ and Masogo $(\mathrm{RR}=4.38,95 \% \mathrm{Cl} 1.62-11.80, P=0.004)$ and in sampling An. arabiensis in Masogo $(R R=5.37,95 \% \mathrm{Cl} 2.17-13.24, P<0.001)$. OLT sampled significantly higher numbers of An. coustani in Kakola Ombaka ( $R R=3.03,95 \% \mathrm{Cl} 1.65-5.56, P<0.001)$ and Masogo $(R R=2.88,95 \% \mathrm{Cl} 1.15-7.22$, $P=0.02)$ compared to HLC. OLT, HLC and MET sampled mostly An. coustani, FTT had similar proportions of An. funestus and An. arabiensis, while HDT sampled predominantly An. arabiensis in both villages. FTT showed close correlation with ILT in vector abundance for all three species at both collection sites.

Conclusion: FTT and OLT are simple, easily scalable traps and are potential replacements for HLC in outdoor sampling of Anopheles mosquitoes. However, the FTT closely mirrored indoor CDC light trap in mosquito indices and therefore may be more of an indoor mimic than a true outdoor collection tool. HDT and MET show potential for sampling outdoor host-seeking mosquitoes. However, the traps as currently designed may not be feasible for large-scale, longitudinal entomological monitoring. Therefore, the baited outdoor CDC light trap may be the most appropriate tool currently available for assessment of outdoor-biting and malaria transmission risk.
\end{abstract}

*Correspondence: abongoben@gmail.com

${ }^{1}$ Centre for Global Health Research, Kenya Medical Research Institute, P.O.

Box 1578, Kisumu, Kenya

Full list of author information is available at the end of the article

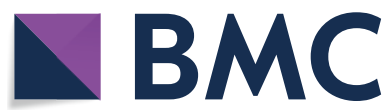

(c) The Author(s) 2021. This article is licensed under a Creative Commons Attribution 4.0 International License, which permits use, sharing, adaptation, distribution and reproduction in any medium or format, as long as you give appropriate credit to the original author(s) and the source, provide a link to the Creative Commons licence, and indicate if changes were made. The images or other third party material in this article are included in the article's Creative Commons licence, unless indicated otherwise in a credit line to the material. If material is not included in the article's Creative Commons licence and your intended use is not permitted by statutory regulation or exceeds the permitted use, you will need to obtain permission directly from the copyright holder. To view a copy of this licence, visit http://creativeco mmons.org/licenses/by/4.0/. The Creative Commons Public Domain Dedication waiver (http://creativecommons.org/publicdomain/ zero/1.0/) applies to the data made available in this article, unless otherwise stated in a credit line to the data. 


\section{Introduction}

Anopheles funestus and An. gambiae/An. coluzzii are considered to be the most efficient malaria vectors in sub-Saharan Africa, largely due to their high levels of anthropophagy [1]. The endophagic and endophilic nature of these primary malaria vector species has led to the use of widespread indoor focused interventions including insecticide treated nets (ITNs) and indoor residual spraying (IRS). The implementation of these measures has contributed to substantial reductions in the malaria burden in sub-Saharan Africa since 2000 [2, $3]$. However, sustained indoor targeted control measures have been associated with changes in malaria vector species composition [4-6] and possibly behaviour [7-9]. In some settings, these changes have been associated with increased proportions of more exophilic vector species such as An. arabiensis or changes in the biting and resting behaviour of An. gambiae or $A n$. funestus, leading to relative increases in outdoor transmission [10-13]. Sustained indoor vector control through ITNs and IRS in western Kenya has resulted in changes in species composition, with the more generalist species $A n$. arabiensis becoming the predominant species in some locations [4, 7] and changes in An. gambiae (s.s.) behaviour resulting in earlier biting [9] with a high frequency of animal and mixed human-animal blood meals [8]. Longitudinal monitoring of vector species composition, biting rates, physiological status, biting and resting behaviour, and ecology is fundamental to understanding the risk of malaria transmission in an area, identifying future threats and formulating methods of control and monitoring [14]. However, outdoor biting mosquitoes pose new challenges in sub-Saharan Africa as they cannot be reliably monitored using many of the current collection methods and tools to control them effectively are lacking.

When conducted under close supervision, the human landing catch (HLC) is generally considered the gold standard for indoor and outdoor collections which can provide critical information on malaria vector species composition, biting densities and time of biting [15]. However, HLC is a labour-intensive and costly procedure requiring trained collectors and extensive supervision $[16,17]$ and is unsustainable for largescale operational sampling of malaria vectors. There are also ethical questions regarding the use of human collectors to attract pathogen transmitting mosquitoes $[18,19]$. While provision of malaria chemoprophylaxis has been demonstrated to be protective to HLC collectors [20], there is still potential for transmission of arboviruses and other mosquito-transmitted pathogens [18]. Due to these ethical concerns coupled with logistical challenges in HLC implementation, the technique is rarely used for routine monitoring of mosquito populations.

Routine monitoring of indoor mosquito populations in western Kenya has been conducted using indoor Centers for Disease Control and Prevention light trap (ILT) and pyrethrum spray catch (PSC). The ILT has been observed by some studies to be an effective alternative to indoor HLC [21-24], whereas other studies have reported the trap to collect fewer Anopheles than HLC [25]. Mathenge et al. (2005) showed that ILT collected $60 \%$ of An. arabiensis and $120 \%$ of An. funestus compared to indoor HLC [24]. Pyrethrum spray catch (PSC) and ILT have become standard trapping methods to monitor indoor populations, but no reliable method has emerged to replace HLC outdoors. As a result, longitudinal entomological monitoring conducted by the President's Malaria Initiative (PMI) VectorLink Project from 2017 to 2018 consisted only of indoor trapping using ILT, PSC and use of window exit traps, with no routine outdoor trapping taking place.

Scalable traps designed specifically for monitoring outdoor biting and resting malaria vectors are urgently needed [12]. The choice of collection method for operational surveillance should be driven by trap efficacy and scalability rather than fine-scale precision with respect to human landing catches (HLC) [23]. This study compared four outdoor traps with HLC as a positive control to identify a suitable replacement for the HLC for longitudinal surveillance of outdoor biting malaria vectors. The primary objective was to determine outdoor trap efficacy for estimating Anopheles numbers per trapping night in comparison with the 'gold standard' HLC. Secondary objectives were to determine the outdoor biting mosquito species composition and to determine endophily proportion by comparing each outdoor trap with indoor CDC light trap (ILT).

\section{Methods \\ Study sites}

The study was conducted in May/June 2018 in two villages, Kakola Ombaka $\left(0.25^{\circ} \mathrm{S}, 34.88^{\circ} \mathrm{E}\right)$ near the Ahero rice irrigation scheme in Nyando sub-county and Masogo village $\left(0.16^{\circ} \mathrm{S}, 35.19^{\circ} \mathrm{E}\right)$ in Mhoroni sub-county, Kisumu County, western Kenya (Fig. 1). The villages were selected to represent areas with high (Kakola Ombaka) and low (Masogo) mosquito densities. The study was conducted in the months of May and June coinciding with the end of the main rainy season and the period of peak mosquito numbers and malaria transmission. 


\section{Outdoor trapping methods}

Four trapping methods-Furvela tent-trap Mk 1.1 (FTT), host decoy trap (HDT), mosquito electrocuting trap (MET) and outdoor CDC-LT (OLT)-were compared with the human landing catch (HLC) in a $5 \times 5$ replicated Latin square experimental design of trapping methods $\times$ housing compounds (Additional file 1: Table S1). The study was conducted for 25 nights in five compounds per night in each village. Distance between the trapping compounds was approximately $100 \mathrm{~m}$. Traps were rotated between the housing compounds nightly, so that over five trapping nights each trap was rotated between all five house compounds. Collectors worked in two shifts each night and were rotated weekly between collection locations each night. Housing compounds where outdoor traps were set were at least $100 \mathrm{~m}$ from each other to minimize the risk of host-odour interference. The location for each outdoor collection method was marked in each compound to ensure consistency throughout the study. Outdoor collections were made approximately 5-10 $\mathrm{m}$ away from the house in a cleared space. In each housing compound where outdoor trapping was conducted, an indoor CDC light trap (ILT) was set inside the house each night for comparison with the outdoor catch. The ILT was installed in a bedroom at approximately

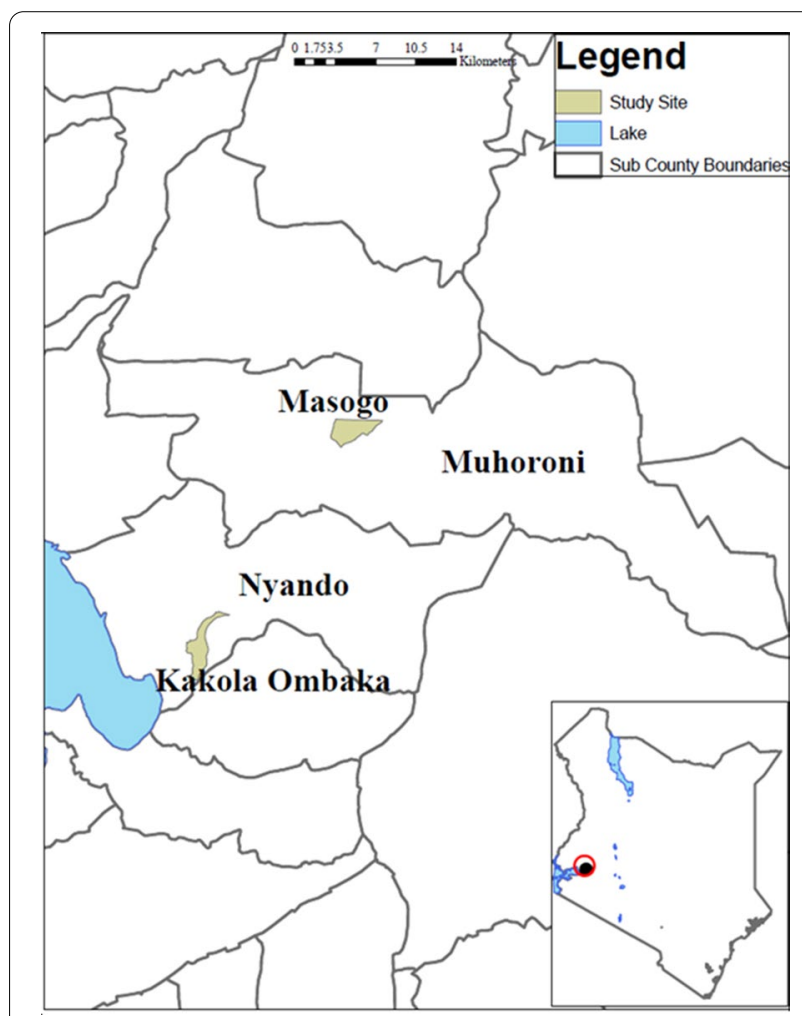

Fig. 1 Map of the study area
$1.5 \mathrm{~m}$ above the floor next to an occupied in situ ITN. All collections were performed from 18:00 to 07:00 the following morning. Figure 2 shows photographs of the five outdoor trapping methods. These are described in more detail below:

\section{Furvela tent trap}

The Furvela tent trap was developed and tested in the village of Furvela, Mozambique, and has been utilized in several locations including Tanzania, Ghana and Cambodia [26-28]. The basic principle of the Furvela tent trap is that human odour and exhaled gases emanating from a gap, the diameter of a CDC trap, in the predominantly closed door of the tent, attract mosquitoes to the gap on the tent door. Close to the gap, the fan and collection bag of a CDC trap (without the light, lid or grid) are placed horizontally outside the tent, 2 to $3 \mathrm{~cm}$ from the opening in the door. On approach to the opening, the insects are sucked into the trap and held in the trap collection bag (Fig. 2B1-2). The suction from the fan effectively prevents any mosquitoes from entering the tent, even at high densities, so that the sleeper is not exposed to biting risk. Mosquitoes are removed from the collection bag using a mouth aspirator and transferred to a holding cup.

\section{Host decoy trap}

The Host Decoy Trap (HDT) was first evaluated in the field in 2015 in Burkina Faso. The HDT exploits the blood-seeking behaviour of mosquitoes by mimicking the sensory stimuli that a mosquito follows when searching for a person to bite. These include host odour, a visual stimulus and body temperature of warm-blooded hosts. These stimuli are incorporated into a trap that lures mosquitoes towards it and then captures them when they land. The trap was set as described by Hawkes et al. [29]. Briefly, the host decoy trap is a cylindrical container filled with warm water, insulated with Styrofoam to prevent heat loss and regulate the surface temperature. The container is covered with a black jacket to provide visual contrast and transparent sticky tape to which mosquitoes get stuck on landing. Host odour from a nearby occupied tent is exhausted using a fan, pushed through a pipe and vented close to the trap (Fig. 2C1-2). Mosquitoes attracted to an odour source are induced to land upon the visually conspicuous, warm trap, where upon they get stuck. The mosquitoes are recovered from the trap by dissolving the glue upon which they are stuck [29].

\section{Electrocuting grids}

These devices were originally developed to quantify the numbers of tsetse flies attracted to humans and wildlife hosts by placing electrocuting nets in an incomplete ring around the host species [30]. The electrocuting grid 

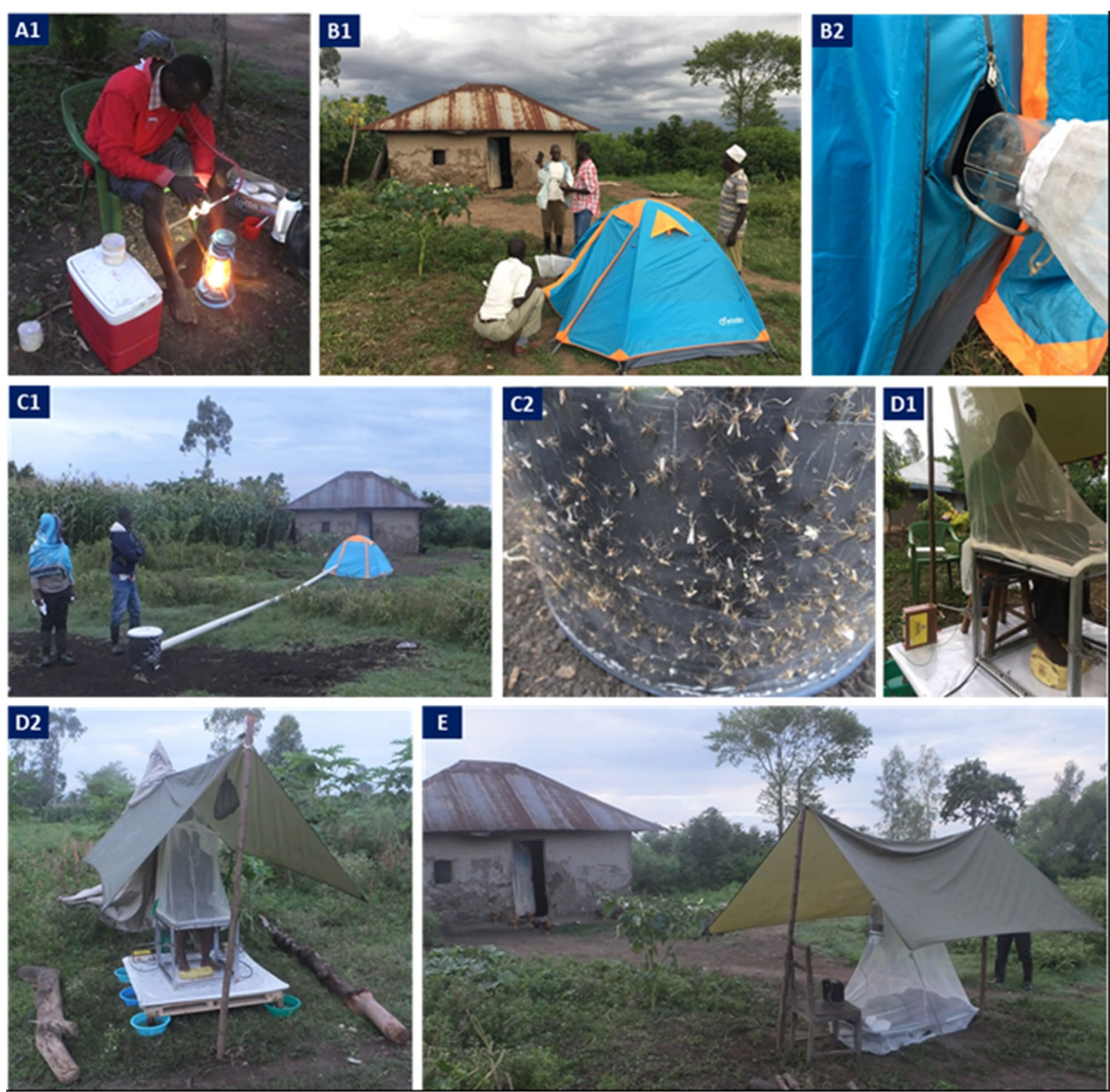

E

Fig. 2 Photographs showing the five outdoor trapping methods that were compared. A1 Human landing catch (HLC), B1 Furvela tent trap (FT B2 FTT opening with CDC light trap (light removed) attached, C1 human decoy trap (HDT), showing tube taking human odour to the heated cylinder sticky trap, C2 HDT, showing mosquitoes stuck to sticky panel on heated cylinder, D1, 2 electrocuting grid trap (EGT), E outdoor CDC light trap (CDC LT), hung next to human sleeping inside an untreated bed net

is effectively invisible to flying insects which, as they approach a stimulus such as a vertebrate host, inadvertently collide with it and are either killed or stunned. Electrocuting grids ( $0.5 \mathrm{~m}$ high; $1 \mathrm{~m}$ wide) consisted of vertical copper wires, $0.2 \mathrm{~mm}$ in diameter, $5 \mathrm{~mm}$ apart and spray painted black to reduce their visibility to flying insects. Alternate wires were earthed or charged by a transformer with a direct current (DC) input (12 V: $3 \mathrm{~A})$ and an output of $50 \mathrm{kV}$, pulsing at $\sim 70 \mathrm{~Hz}$. Insects killed or stunned after colliding with the grids were collected on a sticky panel placed under the electrocuting grid. A simple shelter was erected over the collector to protect them from the rain. The collector sat on a stool and four panels of electrocuting grids were arranged around the legs up to the knee level. The rest of the body was covered with an untreated bed net attached to the top frame 
of the electrocuting grid. Mosquitoes attempting to access the collector were electrocuted and dropped on the sticky panel under the grid from where they were collected and recovered by dissolving the glue (Fig. 2D1-2).

\section{CDC light trap (outdoors)}

CDC light traps are battery powered with a motorized fan, light bulb and a mosquito collection cup. The trap can be used with $\mathrm{CO}_{2}$ to mimic breath exhalation. Mosquitoes attracted to the traps by either light and/or $\mathrm{CO}_{2}$ are drawn in at the top and forced downward by the fan into the collection cup, from which they cannot escape. Malaria-transmitting mosquitoes are nocturnal; therefore, traps are typically deployed at dusk and collected at dawn the following day. When deployed indoors, the optimum location for sampling house-visiting mosquitoes has been reported to be as close as possible to the host, with improved catching efficiency when the trap is installed at the foot of an occupied untreated bednet [31, 32]. From an epidemiological point of view, the use of a light trap next to an occupied bednet is more meaningful than using an unbaited light trap [33] as it provides a good measure of host-seeking mosquito densities. Outdoor deployment of light traps is not commonly used in surveillance particularly in regions where people are mostly indoors at night. The CDC-LT was hung outdoors at $1.5 \mathrm{~m}$ above the ground next to an occupied, untreated bed net (Fig. 2E). The bed and light trap were protected from rainfall by a tarpaulin.

\section{Human landing catch (outdoors)}

One collector sat outside on a chair with lower limbs exposed and a dimly lit kerosene lamp nearby to provide some light. A torch (flashlight) was used to spot the mosquitoes landing on the exposed lower limbs of the HLC collector. Mosquitoes landing on the exposed limbs were aspirated and transferred into paper cups labelled with the hour of collection (Fig. 3, panel A1). Within each collection hour, the collectors worked for $45 \mathrm{~min}$ with 15 min breaks between the hours and switched shifts at midnight. Collectors provided written consent to participate in the study. They were tested for malaria infection using a malaria rapid diagnostic test 7 days before collections began and all tested negative. The collectors were placed on weekly malaria prophylaxis beginning 7 days before collections began and continuing up to 4 weeks after the end of collections. Over the same period, the collectors were monitored for malaria infection. None tested positive during and up to 4 weeks after HLCs were conducted. Each collector was compensated for time spent in mosquito collection.

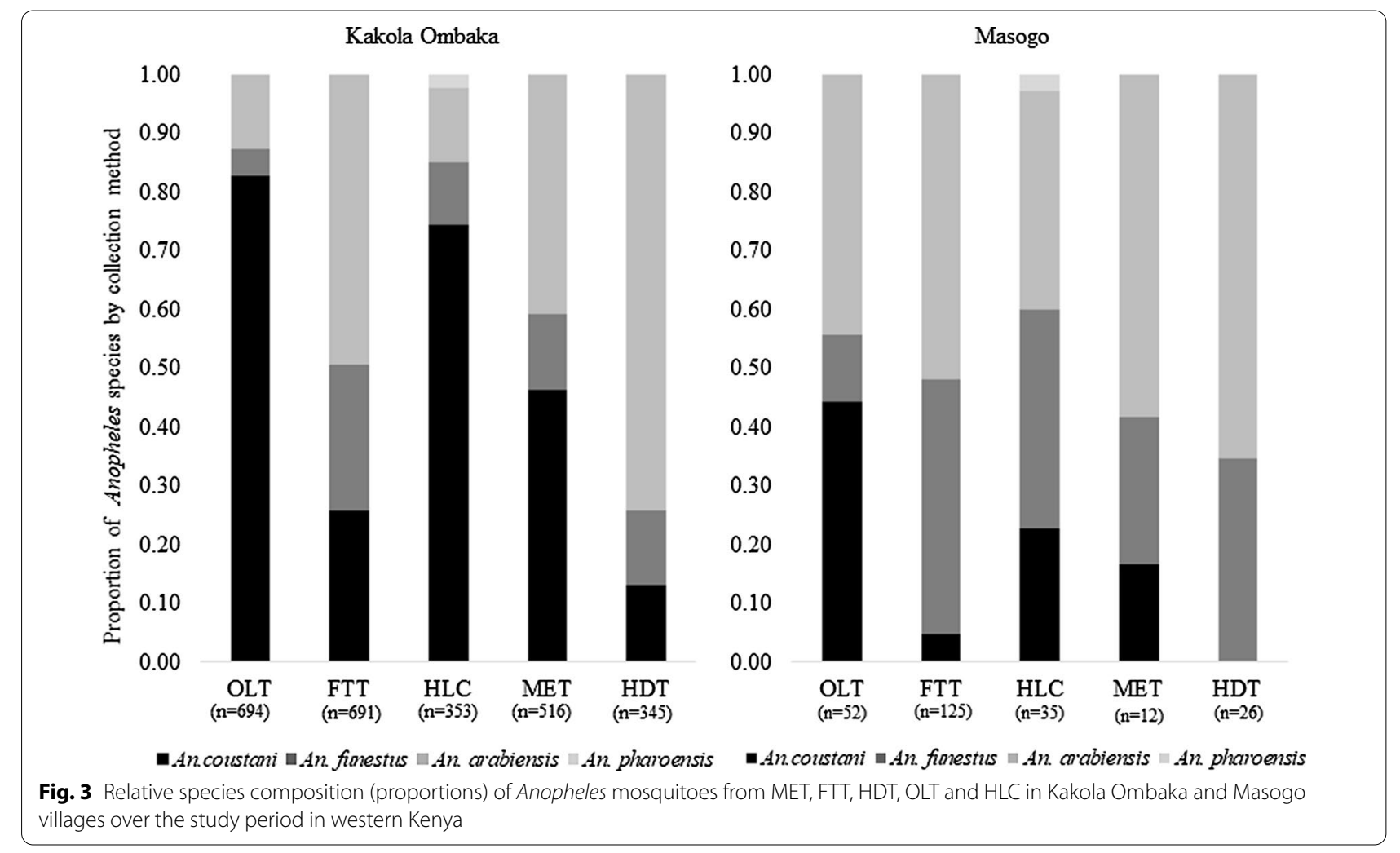




\section{Additional non-Latin square human landing catch}

To assess malaria vector biting behaviour, Plasmodium falciparum infection rates and risk of human to exposure to mosquito bites, an additional 5 nights of HLCs were conducted indoors and outdoors in five houses in Kakola Ombaka. The collections were performed in the last of week of the Latin square outdoor trap comparison study. In each collection house, four collectors worked in pairs in two shifts over the collection period. In each shift, one of the collectors worked outdoors within $5 \mathrm{~m}$ of the house while the other was indoors in the unoccupied living room. The collectors were rotated between the collection shifts and location. The HLC collectors also recorded the location of household members (indoors or outdoors) every hour to assess their risk of exposure to mosquito bites.

\section{Laboratory analysis}

All collected Anopheles were identified morphologically using the keys of Gillies and Coetzee, 1987 [34]. Further species discrimination was performed only for An. gambiae (s.l.) and An. funestus groups while all females were analysed for sporozoite infection. Female mosquitoes were dissected into parts for various procedures: heads and thoraces were used for determination of $P$. falciparum sporozoite infection by enzyme-linked immunosorbent assay (ELISA) using standard operating procedures as described in the MR4 Methods in Anopheles Research [35], adapted from Wirtz et al. [36]. The legs and wings were used in PCR analyses to identify to species level members of the An. gambiae species complex and Anopheles funestus group [35]. The protocol of Scott et al. [37] as described in standard operating procedures in the MR4 Methods in Anopheles Research [35] was used for distinguishing between different species of An. gambiae while the protocol of Koekemoer et al. [38] was used to identify members of the An. funestus species group.

\section{Data analysis}

Analysis was done using $\mathrm{R}$ statistical software version 4.0.2. Data were fitted using Generalized Linear Mixed Effects Models (GLMMs) to describe how well the other traps proportionately sample the same composition and species as HLC. Since the data were over-dispersed, the package glmmADMB [39] was used to fit negative binomial distribution models for the analysis of mosquito numbers in the outdoor traps for the Latin square study. The numbers of female Anopheles mosquitoes were assessed as a function of collection method as a fixed effect while collection compounds and days were treated as random factors. The same statistical package was used to assess catch sizes in indoor CDC light traps and each of the paired outdoor traps; the numbers of female Anopheles mosquitoes were assessed as a function of collection method as a fixed effect and collection compound as a random factor. Pairwise comparisons of the mean numbers of each Anopheles species collected by the different trapping methods were done by Tukey's test. To analyse Anopheles species proportions for each trapping method, a binomial GLM model was used.

\section{Results}

Mosquito species composition and abundance

From the Latin square comparison of outdoor traps, a total of 2849 female Anopheles mosquitoes and 20,093 Culex species were collected outdoors at five sampling locations in each of the two sites over 25 trapping nights outdoors. The most abundant of the Anopheles species based on morphological identification was An. coustani (1339, 47.0\% of all Anopheles mosquitoes) followed by An. gambiae (s.l.) (1066, 37.4\%), An. funestus group (435, 15.3\%) and An. pharoensis (9, 0.3\%). Only 11 male Anopheles mosquitoes were sampled over the collection period (Table 1). Of the female An. gambiae (s.l.) from outdoor traps, 563 samples were analysed by PCR for species identification, and of these, 557 (98.9\%) were $A n$. arabiensis and 6 (1.1\%) An. gambiae (s.s.). Of 212 samples morphologically identified as An. funestus group, all were identified by PCR as An. funestus (s.s.).

Collections by indoor CDC light trap at each of the five trapping locations in the two sites yielded a total of 3249 Anopheles mosquitoes and 7550 Culex species. Among the Anopheles mosquitoes, 1343 were An. gambiae (s.l.) (41.3\% of the total Anopheles), 967 were An. coustani (29.8\%), 936 were An. funestus group (28.8\%) and 3 were other Anopheles species (0.1\%) (Table 1). Of $958 \mathrm{An}$. gambiae (s.l.) collected by indoor CDC light trap and identified to species by PCR, An. arabiensis was predominant (99.0\%) with only 10 identified as An. gambaie (s.s.) (1.0\%). Five hundred thirty-three (533) from An. funestus group were analysed by PCR and all were confirmed to be An. funestus (s.s.). Hereafter, all An. gambiae (s.l.) are assumed to be An. arabiensis and all An. funestus (s.l.) to be An. funestus (s.s.).

Figure 3 shows percentage of Anopheles species composition by trapping method for both the Kakola Ombaka and Masogo sites. The Anopheles species composition differed by trapping method in both sites. Anopheles coustani was the predominant species collected by OLT and HLC in Kakola Ombaka village, accounting for $82.9 \%$ of Anopheles by OLT and $74.5 \%$ by HLC. An. arabiensis was predominant in HDT and FTT, accounting for $74.2 \%$ of Anopheles in HDT and 49.4\% in FTT. Anopheles funestus was collected in lower proportions in all outdoor traps compared to An. coustani and An. arabiensis. 
Table 1 Numbers of Anopheles and Culex mosquitoes sampled by different collection methods outdoor (Latin square study) and indoor by CDC light traps in Kakola Ombaka and Masogo villages over 25 trapping nights

\begin{tabular}{|c|c|c|c|c|c|c|c|c|c|}
\hline \multirow{2}{*}{$\begin{array}{l}\text { Collection } \\
\text { location }\end{array}$} & \multirow[t]{2}{*}{ Study site } & \multirow{2}{*}{$\begin{array}{l}\text { Collection } \\
\text { method }\end{array}$} & \multicolumn{4}{|c|}{ Female Anopheles } & \multirow{2}{*}{$\begin{array}{l}\text { Total } \\
\text { female } \\
\text { Anopheles }\end{array}$} & \multirow[t]{2}{*}{ Male Anopheles } & \multirow{2}{*}{$\begin{array}{l}\text { Total female } \\
\text { Culex species }\end{array}$} \\
\hline & & & An. funestus & An. arabiensis & An. coustani & An. pharoensis & & & \\
\hline \multirow[t]{11}{*}{ Outdoor } & \multirow[t]{5}{*}{ Kakola Ombaka } & MET & 67 & 210 & 239 & 0 & 516 & 2 & 2845 \\
\hline & & $\mathrm{FTT}$ & 172 & 341 & 178 & 0 & 691 & 2 & 4434 \\
\hline & & HDT & 44 & 256 & 45 & 0 & 345 & 1 & 2666 \\
\hline & & OLT & 30 & 89 & 575 & 0 & 694 & 3 & 3712 \\
\hline & & HLC & 37 & 45 & 263 & 8 & 353 & 1 & 3808 \\
\hline & \multirow[t]{5}{*}{ Masogo } & MET & 3 & 7 & 2 & 0 & 12 & 1 & 409 \\
\hline & & $\mathrm{FTT}$ & 54 & 65 & 6 & 0 & 125 & 0 & 713 \\
\hline & & HDT & 9 & 17 & 0 & 0 & 26 & 0 & 411 \\
\hline & & OLT & 6 & 23 & 23 & 0 & 52 & 0 & 236 \\
\hline & & $\mathrm{HLC}$ & 13 & 13 & 8 & 1 & 35 & 1 & 859 \\
\hline & Total & & 435 & 1066 & 1339 & 9 & 2849 & 11 & 20,093 \\
\hline \multirow[t]{3}{*}{ Indoor (ILT) } & Kakola Ombaka & ILT & 779 & 1176 & 954 & 3 & 2912 & 53 & 6157 \\
\hline & Masogo & ILT & 157 & 167 & 13 & 0 & 337 & 44 & 1393 \\
\hline & Total & & 936 & 1343 & 967 & 3 & 3249 & 97 & 7550 \\
\hline
\end{tabular}

In Masogo village, An. arabiensis was the most common species across all collection methods: $44.2 \%$ of Anopheles mosquitoes in OLT, 52.0\% FTT, 37.1\% HLC, 58.3\% MET and $65.4 \%$ HDT. An. pharoensis were only observed in HLC at both sites, representing 2.3\% of all Anopheles collected in Kakola Ombaka and 2.9\% in Masogo.

\section{Anopheles densities according to trap method}

The mean numbers of Anopheles species collected outdoors by MET, FTT, HDT, OLT and HLC at Kakola Ombaka and Masogo villages are presented in Fig. 4 and Table 2. The mean number of An. funestus per trapping night was significantly higher in FTT compared to HLC in both Kakola Ombaka ( $\mathrm{RR}=5.59,95 \%$ CI 2.49-12.55, $P<0.001)$ and Masogo $(\mathrm{RR}=4.38,95 \% \mathrm{CI} 1.62-11.80$, $P=0.004)$. Significantly fewer $A n$. funestus were collected by MET (0.12) compared to HLC (0.52) in Masogo $(\mathrm{RR}=0.24,95 \% \mathrm{CI} 0.05-1.03, P=0.05)$. For all other traps there was no significant difference in the mean nightly catch of An. funestus between MET, HDT, OLT and HLC in Kakola Ombaka or between HDT, OLT and HLC in Masogo village.

Significantly more An. arabiensis were collected by all the outdoor collection methods compared to HLC in Kakola Ombaka: MET $(\mathrm{RR}=4.67,95 \%$ CI 2.44-8.93, $P<0.001)$, FTT $(\mathrm{RR}=7.58,95 \%$ CI3.98-14.42, $P<0.001)$, HDT $(\mathrm{RR}=5.69,95 \%$ CI 2.98-10.86, $P<0.001)$ and OLT $(\mathrm{RR}=1.98,95 \%$ CI 1.01-3.86, $P=0.05)$. However, in Masogo, significantly higher numbers of An. arabiensis were only observed in FTT compared to HLC $(\mathrm{RR}=5.37$, 95\% CI 2.17-13.24, $P<0.001)$. No significant differences were observed in the catch sizes of $A n$. arabiensis between HLC and any of the other collection methods in Masogo.

Significantly higher numbers of $A n$. coustani were caught by OLT compared to HLC in Kakola Ombaka $(\mathrm{RR}=3.03,95 \% \mathrm{CI} 1.65-5.56, P<0.001)$ and in Masogo village $(\mathrm{RR}=2.88,95 \% \mathrm{CI} 1.15-7.22, P=0.02)$ (Table1). There were significantly fewer An. coustani caught by HDT compared to HLC $(\mathrm{RR}=0.18,95 \%$ CI $0.09-0.37$, $P<0.001)$ in Kakola Ombaka. For all other trapping methods there was no significant difference in An. coustani mean collection densities. Pairwise comparisons of mean densities of different Anopheles species by collection methods in Kakola Ombaka and Masogo are presented in Additional file 1: Tables S2, S3 respectively.

\section{Comparison of outdoor traps with indoor CDC light trap}

We compared each of the outdoor traps with the paired indoor light trap data to assess catch size ratios of $A n$. arabiensis, An. funestus and An. coustani indoors and outdoors in the same house compound (Tables 3 and 4). This comparison is useful in determining the endophilic/ exophilic nature of each species in the area and determining the relative performance of each outdoor trap.

In Kakola Ombaka, three of the outdoor trapping methods, HLC, OLT and HDT, collected significantly fewer An. funestus than the paired indoor ILT collections. The only outdoor trapping method to collect similar numbers of An. funestus as the ILT was the FTT. For $A n$. arabiensis there was generally no significant difference in catch size between indoor light traps 


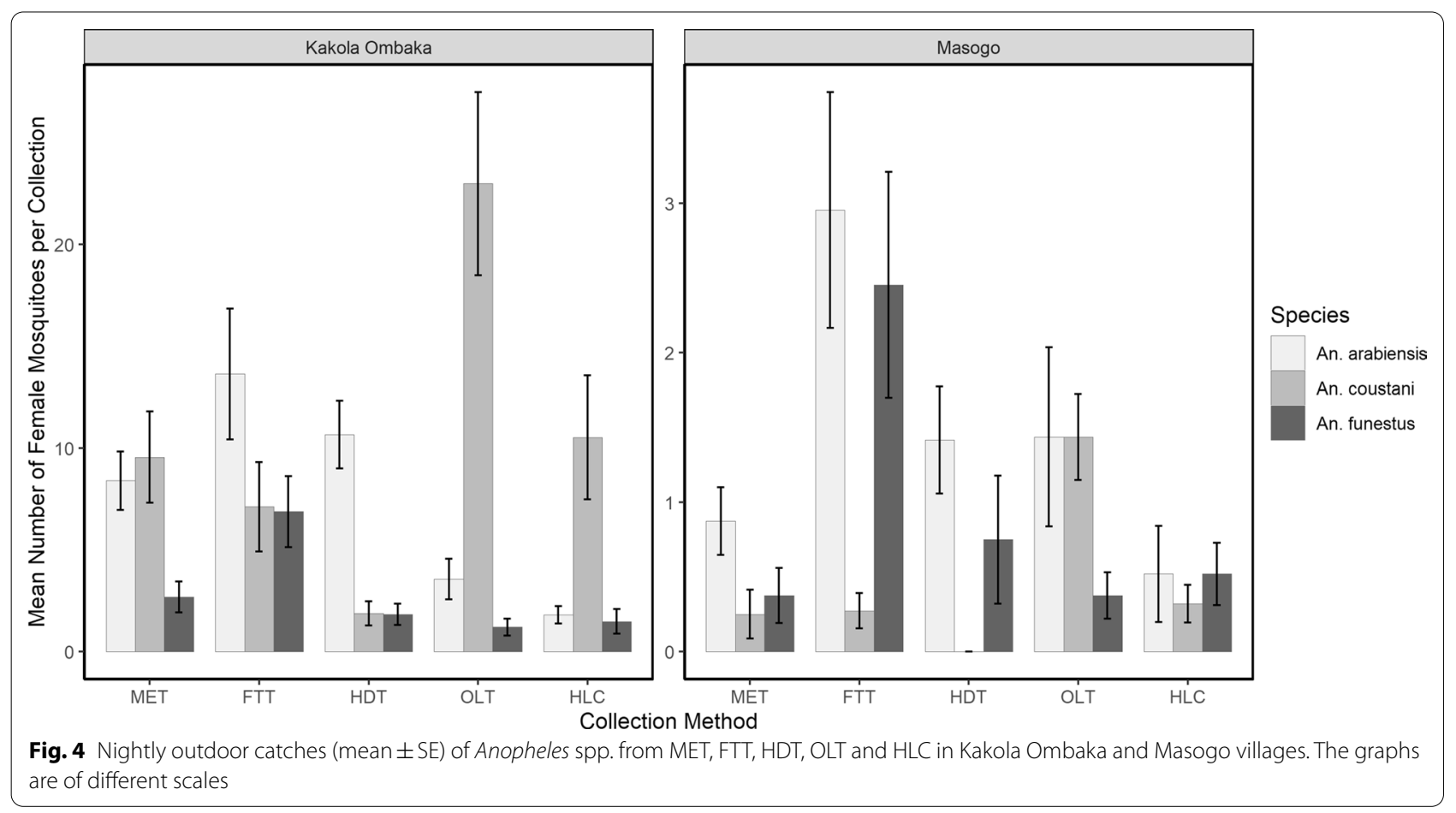

Table 2 Comparison of mean numbers of An. funestus, An. arabiensis and An. coustani collected outdoors by MET, FTT, HDT, OLT and HLC in Kakola Ombaka and Masogo villages over the study period

\begin{tabular}{|c|c|c|c|c|c|c|c|c|c|c|c|}
\hline \multirow[t]{2}{*}{ Category } & \multirow{2}{*}{$\begin{array}{l}\text { Collection } \\
\text { Method }\end{array}$} & \multicolumn{5}{|c|}{ Kakola Ombaka } & \multicolumn{5}{|c|}{ Masogo } \\
\hline & & Mean & Risk ratio & Lower CL & Upper CL & $P$-value & Mean & Risk ratio & Lower CL & Upper CL & $P$-value \\
\hline \multirow[t]{5}{*}{ An. funestus } & MET & 2.68 & 1.98 & 0.88 & 4.46 & 0.1 & 0.12 & 0.24 & 0.05 & 1.03 & 0.05 \\
\hline & $\mathrm{FTT}$ & 6.88 & 5.59 & 2.49 & 12.55 & $<0.001$ & 2.16 & 4.38 & 1.62 & 11.8 & 0.004 \\
\hline & HDT & 1.76 & 1.38 & 0.60 & 3.18 & 0.44 & 0.36 & 0.66 & 0.21 & 2.09 & 0.48 \\
\hline & OLT & 1.20 & 0.88 & 0.37 & 2.11 & 0.78 & 0.24 & 0.45 & 0.13 & 1.57 & 0.21 \\
\hline & $\mathrm{HLC}$ & 1.48 & Ref & & & & 0.52 & Ref & & & \\
\hline \multirow[t]{5}{*}{ An. arabiensis } & MET & 8.40 & 4.67 & 2.44 & 8.93 & $<0.001$ & 0.28 & 0.59 & 0.19 & 1.87 & 0.37 \\
\hline & $\mathrm{FTT}$ & 13.64 & 7.58 & 3.98 & 14.42 & $<0.001$ & 2.26 & 5.37 & 2.17 & 13.24 & $<0.001$ \\
\hline & HDT & 10.24 & 5.69 & 2.98 & 10.86 & $<0.001$ & 0.68 & 1.32 & 0.49 & 3.59 & 0.58 \\
\hline & OLT & 3.56 & 1.98 & 1.01 & 3.86 & 0.05 & 0.92 & 1.83 & 0.70 & 4.79 & 0.22 \\
\hline & $\mathrm{HLC}$ & 1.80 & Ref & & & & 0.52 & Ref & & & \\
\hline \multirow[t]{5}{*}{ An. coustani } & MET & 9.56 & 1.11 & 0.61 & 2.03 & 0.74 & 0.08 & 0.00 & 0.00 & $\operatorname{lnf}$ & 1.00 \\
\hline & $\mathrm{FTT}$ & 7.12 & 0.66 & 0.35 & 1.23 & 0.19 & 0.24 & 7.50 & 0.24 & 2.37 & 0.62 \\
\hline & HDT & 1.80 & 0.18 & 0.09 & 0.37 & $<0.001$ & 0.00 & 0.00 & 0.00 & $\operatorname{lnf}$ & 1.00 \\
\hline & OLT & 23.00 & 3.03 & 1.65 & 5.56 & $<0.001$ & 0.92 & 2.88 & 1.15 & 7.22 & 0.02 \\
\hline & HLC & 10.52 & Ref & & & & 0.32 & Ref & & & \\
\hline
\end{tabular}

The models included terms for collection methods and an interaction term. The risk ratios (RR) were generated by exponentiating the model coefficients

and outdoor methods, with the exception of HLC and OLT in Kakola Ombaka where numbers were significantly lower in both HLC and OLT compared to ILT. For An. coustani, there was no difference in the catch sizes between MET, FTT and HLC outdoors compared to ILT indoors. However, HDT sampled significantly fewer An. coustani while OLT sampled significantly more compared to ILT. In Masogo there were no clear differences although densities were much lower. The only outdoor trapping method to catch a greater number of An. coustani compared to paired ILT was the OLT. For all other methods there was no significant 
Table 3 Comparison of mean numbers of An. funestus, An. arabiensis and An. coustani collected outdoors and indoors between each of the outdoor trapping methods (MET, FTT, HDT, OLT, HLC) and ILT in Kakola Ombaka village

\begin{tabular}{|c|c|c|c|c|c|c|c|}
\hline Comparison & Anopheles species & $\begin{array}{l}\text { Collection } \\
\text { method }\end{array}$ & Mean & Risk ratio & Lower CL & Upper CL & $P$-values \\
\hline \multirow[t]{6}{*}{ MET and ILT } & An. arabiensis & MET & 8.40 & 0.97 & 0.42 & 2.29 & 0.96 \\
\hline & & ILT & 13.08 & Ref & & & \\
\hline & An. funestus & MET & 2.68 & 0.51 & 0.24 & 1.11 & 0.09 \\
\hline & & ILT & 5.28 & Ref & & & \\
\hline & An. coustani & MET & 9.56 & 1.24 & 0.52 & 2.92 & 0.63 \\
\hline & & ILT & 8.16 & Ref & & & \\
\hline \multirow[t]{6}{*}{ FTT and ILT } & An. arabiensis & FTT & 13.64 & 1.36 & 0.73 & 2.56 & 0.33 \\
\hline & & ILT & 10.00 & Ref & & & \\
\hline & An. funestus & FTT & 6.88 & 0.93 & 0.48 & 1.77 & 0.81 \\
\hline & & ILT & 7.96 & Ref & & & \\
\hline & An. coustani & FTT & 7.12 & 0.79 & 0.32 & 1.91 & 0.59 \\
\hline & & ILT & 7.52 & Ref & & & \\
\hline \multirow[t]{6}{*}{ HDT and ILT } & An. arabiensis & HDT & 10.24 & 1.25 & 0.65 & 2.40 & 0.50 \\
\hline & & ILT & 8.44 & Ref & & & \\
\hline & An. funestus & HDT & 1.76 & 0.29 & 0.13 & 0.64 & 0.002 \\
\hline & & $\| \mathrm{LT}$ & 6.24 & Ref & & & \\
\hline & An. coustani & HDT & 1.80 & 0.21 & 0.10 & 0.49 & $<0.001$ \\
\hline & & $\mathrm{ILT}$ & 7.68 & Ref & & & \\
\hline \multirow[t]{6}{*}{ HLC and ILT } & An. arabiensis & $\mathrm{HLC}$ & 1.80 & 0.26 & 0.11 & 0.59 & 0.001 \\
\hline & & ILT & 6.92 & Ref & & & \\
\hline & An. funestus & HLC & 1.48 & 0.25 & 0.09 & 0.65 & 0.005 \\
\hline & & $\mathrm{ILT}$ & 5.92 & Ref & & & \\
\hline & An. coustani & $\mathrm{HLC}$ & 10.52 & 1.24 & 0.54 & 2.90 & 0.60 \\
\hline & & ILT & 7.16 & Ref & & & \\
\hline \multirow[t]{6}{*}{ OLT and ILT } & An. arabiensis & OLT & 3.56 & 0.42 & 0.20 & 0.89 & 0.03 \\
\hline & & ILT & 8.60 & Ref & & 1 & 1 \\
\hline & An. funestus & OLT & 1.20 & 0.21 & 0.09 & 0.81 & $<0.001$ \\
\hline & & ILT & 5.76 & Ref & & & \\
\hline & An. coustani & OLT & 23.00 & 3.00 & 1.67 & 5.39 & $<0.001$ \\
\hline & & $\mathrm{ILT}$ & 7.64 & Ref & & & \\
\hline
\end{tabular}

The models included terms for collection methods and an interaction term. The risk ratios (RR) were generated by exponentiating the model coefficients

difference compared to ILT, except for the HDT, which caught significantly lower numbers.

Comparison of catch sizes in pairs of indoor and outdoor CDC light traps in Kakola Ombaka showed that significantly lower numbers of $A n$. arabiensis ( $\mathrm{RR}=0.42,95 \% \mathrm{CI} 0.20-0.89, P=0.03)$ and An. funestus $(\mathrm{RR}=0.21,95 \%$ CI $0.09-0.81, P<0.001)$ were collected in OLT compared to indoor ILT whereas significantly higher numbers of An. coustani ( $R \mathrm{R}=3.00,95 \% \mathrm{CI}$ 1.67-5.39, $P<0.001)$ were observed in OLT compared to ILT (Table 3). A similar trend was also recorded in Masogo with fewer An. funestus in OLT than ILT ( $\mathrm{RR}=0.21,95 \%$ CI $0.08-0.57, P=0.002)$ but more $A n$. coustani captured in OLT than ILT $(\mathrm{RR}=5.75,95 \% \mathrm{CI}$ 1.79-18.46, $P=0.003$ ) (Table 4).
Significantly lower numbers of An. arabiensis (RR $=0.26,95 \% \mathrm{CI} 0.11-0.59, P=0.001)$ and An. funestus $(\mathrm{RR}=0.25,95 \% \mathrm{CI} 0.09-0.65, P=0.005)$ were collected by HLC outdoors compared to ILT in Kakola Ombaka. There was no significant difference in the numbers of An. coustani collected by these two methods. In Masogo, there was no significant difference in the catch sizes between the two HLC and ILT for all the three Anopheles species. There was no significant difference in the numbers of An. arabiensis, An. funestus and An. coustani sampled outdoors by FTT compared to paired ILT collections in Kakola Ombaka or Masogo $(P>0.05)$. 
Table 4 Comparison of mean numbers of An. funestus, An. arabiensis and An. coustani collected outdoors and indoor between each of the outdoor trapping methods (MET, FTT, HDT, OLT, HLC) and ILT in Masogo village

\begin{tabular}{|c|c|c|c|c|c|c|c|}
\hline Comparison & Anopheles species & $\begin{array}{l}\text { Collection } \\
\text { method }\end{array}$ & Mean & Risk ratio & Lower CL & Upper CL & $P$-values \\
\hline \multirow[t]{6}{*}{ MET and ILT } & An. arabiensis & MET & 0.28 & 0.18 & 0.06 & 0.48 & 0.001 \\
\hline & & ILT & 1.44 & Ref & & & \\
\hline & An. funestus & MET & 0.12 & 0.14 & 0.03 & 0.54 & 0.005 \\
\hline & & ILT & 0.88 & Ref & & & \\
\hline & An. coustani & MET & 0.08 & 2.00 & 0.18 & 22.06 & 0.57 \\
\hline & & ILT & 0.04 & Ref & & & \\
\hline \multirow[t]{6}{*}{ FTT and ILT } & An. arabiensis & FTT & 2.60 & 1.87 & 0.94 & 3.72 & 0.07 \\
\hline & & ILT & 1.48 & Ref & & & \\
\hline & An. funestus & FTT & 2.16 & 1.20 & 0.57 & 2.53 & 0.64 \\
\hline & & ILT & 1.80 & Ref & & & \\
\hline & An. coustani & FTT & 0.24 & 3.00 & 0.58 & 15.39 & 0.19 \\
\hline & & ILT & 0.08 & Ref & & & \\
\hline \multirow[t]{6}{*}{ HDT and ILT } & An. arabiensis & HDT & 0.68 & 0.50 & 0.23 & 1.08 & 0.08 \\
\hline & & $\mathrm{ILT}$ & 1.48 & Ref & & & \\
\hline & An. funestus & HDT & 0.36 & 0.28 & 0.09 & 0.80 & 0.02 \\
\hline & & ILT & 1.36 & Ref & & & \\
\hline & An. coustani & HDT & 0.00 & 0.00 & 0.00 & $\operatorname{lnf}$ & 1.00 \\
\hline & & ILT & 0.16 & Ref & & & \\
\hline \multirow[t]{6}{*}{ HLC and ILT } & An. arabiensis & $\mathrm{HLC}$ & 0.52 & 0.45 & 0.17 & 1.20 & 0.11 \\
\hline & & ILT & 1.08 & Ref & & & \\
\hline & An. funestus & $\mathrm{HLC}$ & 0.52 & 0.48 & 0.15 & 1.52 & 0.21 \\
\hline & & ILT & 1.08 & Ref & & & \\
\hline & An. coustani & $\mathrm{HLC}$ & 0.32 & 4 & 0.80 & 20.10 & 0.09 \\
\hline & & ILT & 0.08 & Ref & & & \\
\hline \multirow[t]{6}{*}{ OLT and ILT } & An. arabiensis & OLT & 0.92 & 0.74 & 0.30 & 1.84 & 0.52 \\
\hline & & ILT & 1.20 & Ref & & & \\
\hline & An. funestus & OLT & 0.24 & 0.21 & 0.08 & 0.57 & 0.002 \\
\hline & & ILT & 1.16 & Ref & & & \\
\hline & An. coustani & OLT & 0.92 & 5.75 & 1.79 & 18.46 & 0.003 \\
\hline & & ILT & 0.16 & Ref & & & \\
\hline
\end{tabular}

The models included terms for collection methods and an interaction term. The risk ratios (RR) were generated by exponentiating the model coefficients

\section{Additional non-Latin square indoor and outdoor HLC collections}

A total of 1689 female Anopheles mosquitoes were collected by HLC indoors and outdoors at five trapping locations over 5 nights. Of the total collections, 1492 (88.3\%) were An. funestus, 109 (6.5\%) An. arabiensis, 81 (4.8\%) An. coustani and 7 (0.4\%) other species of Anopheles. From indoor HLC, An. funestus was the predominant species, accounting for $93.4 \%$, while An. arabiensis was $4.6 \%$ and An. coustani $1.7 \%$. Similarly, from outdoor HLC, An. funestus accounted for $47.1 \%$, while An. arabiensis was 20.9\%, An. coustani $29.3 \%$ and other Anopheles 2.6\% (Fig. 5).

We estimated the risk of human exposure to mosquito bites at one of the study villages, Kakola Ombaka. Figure 6 shows the number of bites per person per hour by An. funestus and An. arabiensis, adjusted by location of members of the household over the collection period to demonstrate the risk of exposure to mosquito bites. The risk of exposure to bites by An. funestus was highest indoors with peak at approximately nine bites per person occurring between 5:00 a.m. and 6:00 a.m., corresponding to the time when most people left their bedrooms (Additional file 2: Fig. S1) and were no longer protected by ITNs. Outdoor exposure to bites by An. funestus was mostly low with a peak of approximately two bites per person per night occurring between 6:00 a.m. and 7:00 a.m. Exposure occurring indoors in the living room while unprotected by ITNs was estimated to be less than a single bite per person per hour for most of the collection period with a peak 


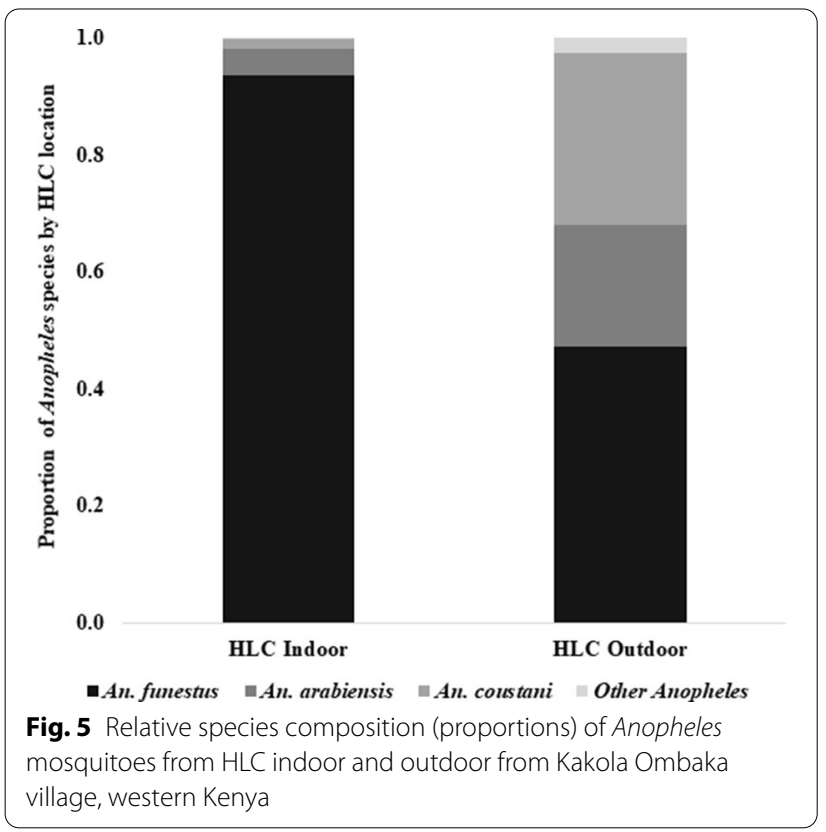

risk of exposure occurring between 6:00 a.m. and 8:00 a.m. Extended exposure to An. funestus bites, albeit at low rates, was observed indoors until 11:00 a.m. when collections ceased (Fig. 6A).

The risk of exposure to bites by An. arabiensis was low compared to that of An. funestus, with a peak of less than a single bite per person per hour. Like biting by An. funestus, the peak biting by An. arabiensis occurred between 5:00 a.m. and 6:00 a.m., corresponding to the time when most people left their bedroom. Risk of exposure to bites by An. arabiensis outdoors was negligible (Fig. 6B).

An assessment of the location of members of the households showed, on average, $78 \%$ of individuals were outdoors between 5:00 p.m and 8:00 p.m. The proportion of people outdoors decreased steadily with a proportionate increase in numbers indoor over time. The proportion of people indoors, in the bedroom area was on average $83 \%$ between 8:00 p.m. and 6:00 a.m. and this decreased steadily between 6:00 a.m. and 7:00 a.m. with an increase in the numbers outdoors over the same time. On average, $69 \%$ of the people were outdoors between $6: 00 \mathrm{a}$.m. and 11:00 a.m. when collection ceased (Additional file 2: Fig. S1).

\section{Plasmodium falciparum sporozoite rates}

A sub-sample of 1253 Anopheles from outdoor traps and 1989 from indoor CDC light traps were analysed for sporozoite infection. From outdoor collections, the overall sporozoite infection rate was $0.4 \%(5 / 1253)$ with infection rates of $1.8 \%$ (4/221) in An. funestus and $0.2 \%$ in (1/599) An. arabiensis. From indoor CDC light trap collections, the overall sporozoite positivity rate was $1.0 \%$ (19/1989) with infection rates of $3.2 \%(17 / 538)$ in $A n$. funestus and $0.2 \%(2 / 961)$ in An. arabiensis. No other species tested positive for sporozoites except An. funestus and An. arabiensis.

\section{Discussion}

This study demonstrated marked differences in Anopheles catch sizes by species in outdoor traps compared to HLC. HLC was considered the gold standard for this

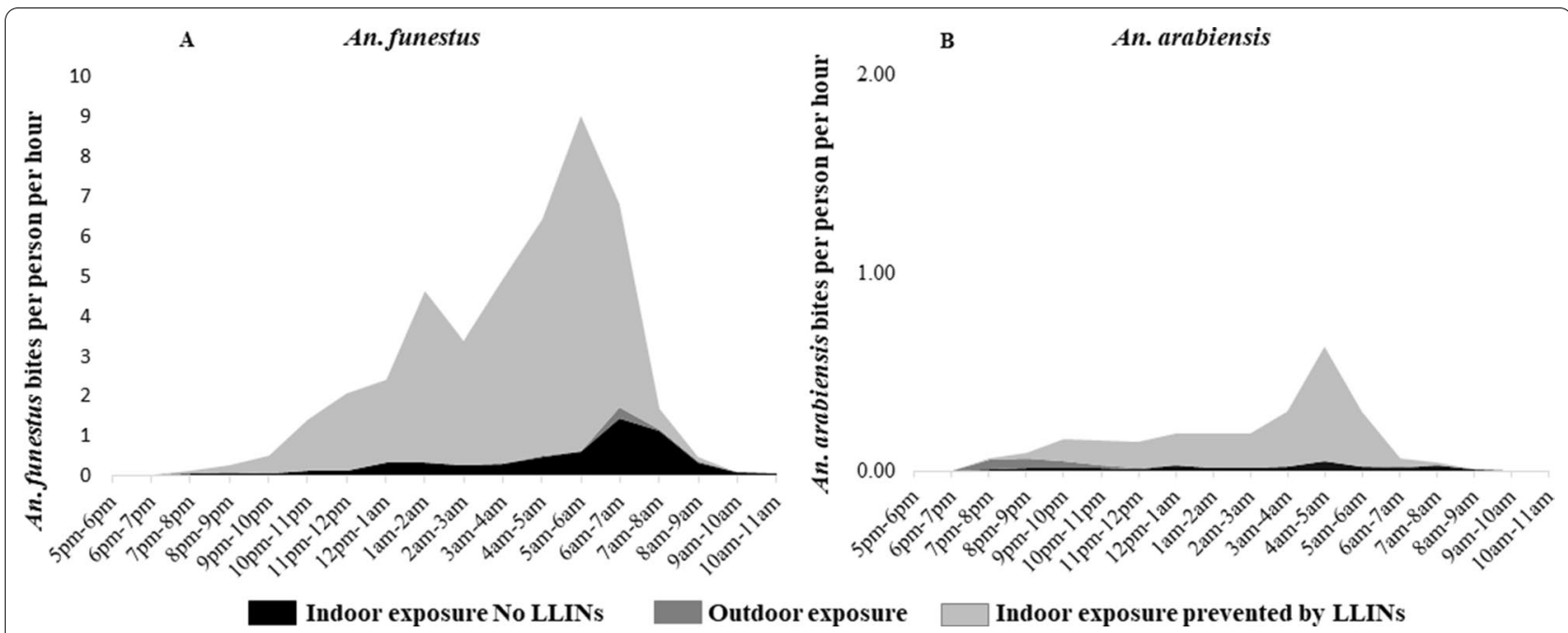

Fig. 6 Profiles of biting by An. funestus (A) and An. arabiensis (B) experienced by the human population in Kakola Ombaka village, westen Kenya. The black area represents exposure that occurs outdoors, the red represents exposure that occurs indoors not prevented by LLINs and the grey represents exposure prevented by LLINS 
study and the prediction was that all other outdoor traps would collect fewer Anopheles than HLC as has been reported in several studies comparing CDC LT indoors with HLC [25]. However, in Kakola Ombaka, HLC captured the fewest An. arabiensis, with all other outdoor traps catching greater densities. The ideal alternative to the HLC either indoors or outdoors should collect mosquitoes in densities that are consistently proportional to the HLC through a range of transmission intensities with similar species compositions. In addition, factors such as cost, feasibility and scalability should be considered in selecting a collection method for operational surveillance [23].

The Furvela tent trap has been described as a simple and effective way of collecting outdoor host-seeking mosquitoes [26]. Similarly, we observed the trap to be a simple tool for sampling mosquitoes that is easy to set up while being comfortable and safe from biting for the person occupying the tent. If the objective is to catch the greatest number of malaria vectors outdoors, the FTT was the most effective in sampling both An. funestus and An. arabiensis outdoors in the two villages. The trap sampled approximately six times more An. funestus compared to HLC in Kakola Ombaka and two times more in Masogo, while the catch size for An. arabiensis was approximately eight and five times more than HLC in the two sites respectively. Both An. funestus and An. arabiensis were caught in the FTT at nearly equal proportions, with relatively low numbers of An. coustani collected in the trap. An important consideration is whether the FTT is truly collecting outdoor host-seeking mosquitoes or is merely a scale model of an indoor environment that traps mosquitoes attempting to enter the tent as they would enter a house. Charlwood et al. concluded that the FTT was truly collecting outdoor biting species in Tanzania and Mozambique, based on the greater diversity of species (such as Mansonia and Coquillettidia spp.) compared to ILT and the greater densities of endophilic species such as An. funestus by ILT [26]. In this study we observed FTT outdoors closely mirrored ILT in both abundance and Anopheles species composition, with no significant difference in An. funestus, An. arabiensis or An. coustani densities between FTT and ILT. In contrast, the ILT densities of An. funestus were greater than all other outdoor trapping methods, including HLC. From this we conclude that An. funestus in the study site were predominantly endophilic, but the FTT mimicked indoor collections, capturing otherwise endophilic mosquitoes as they attempted to enter the tent. In an earlier trap comparison study in western Kenya, the Ifakara tent trap (another tent trap not used in the current investigation) was observed to be the only trap with the numbers of An. funestus captured not significantly different from
HLC indoor [23]. These observations suggest that tent traps such as FTT are not truly outdoor trapping tools but mimics of an indoor environment where the trapped mosquito species exhibit house entry traits.

CDC light traps are commonly installed indoors adjacent to an occupied bed net [31,32]. Outdoor implementation is more challenging, especially in regions where people do not routinely sleep outdoors, including the current study site. Collectors were reluctant to sleep outdoors because of possible security risks from humans and nocturnal animals and the relatively cold weather. OLT was the only trap that sampled more An. coustani in both villages compared to HLC. We conclude that the high densities of An. coustani were partly due to the exophilic nature of this species based on higher densities compared to ILT and partly due to attraction to the light bulb based on the higher densities compared to other outdoor traps. Compared with ILT, the OLT better sampled exophilic and exophagic An. arabiensis and An. coustani whilst the converse was true for An. funestus. Therefore, we conclude that the outdoor CDC light trap was the most useful outdoor trap tested in terms of determining outdoor host-seeking preferences and densities of Anopheles species. The CDC light traps have been reported to be the most effective alternative to HLC [2123] when implemented indoors. Our results demonstrate that implementation of CDC light traps outdoors is similarly an effective alternative to HLC outdoors. However, further investigation is needed to generate data on hourly biting rates compared between CDC light traps and HLC to effectively demonstrate peaks of mosquito biting and exposure.

The host decoy trap was effective in sampling An. arabiensis compared to both HLC and ILT. We have previously demonstrated HDT with cattle odour to be effective for collecting An. arabiensis outdoors in western Kenya, whereas using human odour was not particularly effective [40]. However, in the current study, the HDT trap baited with a human sampled six times more An. arabiensis than HLC. The HDT combines host odours, heat and visual stimuli to simulate a host $[29,40]$, which provides the basis of sampling outdoor host-seeking mosquitoes with a potential of replacing HLC [29]. The trap however requires optimization of the heating system and odour source to ensure consistency and enable scalability and ease of application.

The MET showed promise as an outdoor mosquito trap, with comparable sampling sensitivity to HLC for $A n$. funestus and An. coustani and higher sensitivity in sampling of An. arabiensis at relatively high mosquito densities in Kakola Ombaka. A study in the Kilombero Valley, southern Tanzania, observed the MET to achieve over $50 \%$ sampling sensitivity relative to HLC [41]. A separate 
study in Dar es Salaam investigating an improved prototype of MET observed the trap to be a highly sensitive tool that accurately quantifies epidemiologically relevant metrics of mosquito-biting densities, behaviours and human exposure distribution [42]. Elsewhere, in Burkina Faso, MET was observed to be less sensitive relative to HLC; however, the density of An. gambiae (s.l.) in MET was highly correlated with HLC [43]. We observed MET to correlate well with HLC at relatively high vector numbers but poorly at low mosquito numbers. While a number of studies have reported MET as a safer alternative to HLC for surveillance of mosquitoes outdoors [41-44], we observed the trap to pose an unacceptable level of discomfort to the collectors who were required to sit with minimal body movement, as the trap is located around their legs throughout the collection period.

The poor performance of HLC relative to other trapping methods in sampling outdoor biting mosquitoes may reflect its well-known limitation that accuracy and catch size are reliant on the performance of collectors and performance is difficult to measure. Every effort was made to ensure the quality of HLC collections by working with trained collectors, providing flasks of coffee and conducting nightly supervision. We hypothesize that the numbers of Anopheles mosquitoes collected were lower in HLC because of the large densities of Culex in the area, which may have led HLC collectors to focus on the larger Culex mosquitoes but miss some of the Anopheles. The standardization of other trapping methods is a potential advantage over HLC, which is prone to unknown levels of variation. However, HLC remains the most suitable method for assessing hourly biting behaviour of mosquitoes.

There is a risk of malaria transmission and other infections from both indoor and outdoor mosquito bites. Even though sporozoite infections were highest in indoor collections by CDC light traps, sporozoite-infected mosquitoes were observed outdoors albeit at low proportions. These results confirm the possibility of malaria transmission outdoors away from the protection of indoor based vector control tools. While it is important to monitor outdoor biting of malaria vectors, the level of risk is dependent on human behaviour and the amount of time spent outdoors. In Bioko, despite sustained indoor interventions through LLINs and IRS, fears of high outdoor malaria risk in children proved unfounded as only $4 \%$ of children spent time outside between 22:00 and 05:00 [45]. The risk of Anopheles mosquito bites was estimated based on the location of the study population during HLCs. Human activity outdoors steadily increased from 05:00 a.m. corresponding to the period of increased mosquito biting by both An. funestus and An. arabiensis. These observations indicated that in western Kenya the greatest risk of exposure was indoors late at night but with some outdoor exposure to both species, particularly in the early morning. Consequently, monitoring of outdoor mosquito populations as vector interventions such as ITNs or IRS reduce indoor transmission is critical in understanding the dynamics in malaria transmission.

Like any other study, this evaluation had limitations that merit further investigation. The study was conducted only in the rainy season with peak mosquito density. Therefore, the effect of seasonality on the trapping efficacy of the outdoor mosquito collection methods was not measured. Consequently, further analysis was not conducted to determine if efficacy of the traps was density dependent or not. It may, therefore, be necessary to conduct similar evaluations in both wet and dry seasons and in different ecological settings to measure the trapping efficacy of the various traps in these different scenarios.

\section{Conclusion}

Sampling of outdoor mosquito population is important in understanding changes in the risk of malaria transmission with increased indoor mosquito control efforts. However, data on longitudinal outdoor mosquito monitoring are usually limited because of lack of a safe, efficient, easily deployed outdoor sampling tool. We observed Furvela tent trap to be a simple and effective tool for sampling mosquitoes, easy to set and exposure free with a higher sensitivity relative to HLC in sampling major Anopheles species in western Kenya. However, the trap closely mirrored indoor CDC light trap in mosquito indices and therefore may be more of an indoor mimic than a true outdoor collection tool. Outdoor human-baited CDC light trap on the other had is relatively simple to use and easily scalable and a suitable alternative to HLC with potential for collection of hourly mosquito data. The host decoy trap also demonstrated a potential for sampling outdoor hostseeking mosquitoes with a possibility of replacing HLC. However, the trap needs improvement regarding heating, odour sources and mosquito trapping mechanism to enable scalability. Finally, the MET showed variability in sensitivity relative to HLC at high and low vector densities and may not be easily scalable for routine mosquito collections. Based on these observations, the outdoor light trap is the most appropriate tool currently available for routine assessment of outdoor biting and malaria transmission risk in this setting.

\section{Abbreviations}

HLC: Human landing catch; CDC-LT: CDC light trap; FTT: Furvela tent trap; MET: Mosquito electrocuting trap; HDT: Human decoy trap. 


\section{Supplementary Information}

The online version contains supplementary material available at https://doi. org/10.1186/s13071-021-04794-3.

Additional file 1: Table S1. Schedule showing the rotation of volunteer sleepers and outdoor trapping methods by location $(\mathrm{H} 1-\mathrm{H} 5)$ using a non-random Latin square rotation. Table S2. A pairwise comparison of means of different Anopheles species between collection methods in Kakola Ombaka. Table S3. A pairwise comparison of means of different Anopheles species between collection methods in Masogo.

Additional file 2: Figure S1. Proportion of individuals at different locations (outdoor, living room and bedroom) per collection hour.

\section{Acknowledgements}

We would like to thank the residents of Kakola Ombaka and Masogo villages. Lastly, we thank the PMI-VectorLink Kenya project entomology staff who conducted mosquito collections and sample analysis.

\section{Disclaimer}

The findings and conclusions in this report do not necessarily reflect the official position of the US Department of Health and Human Services or the US Centers for Disease Control and Prevention. The use of trade names is for identification only and does not imply endorsement by the US Department of Health and Human Services or the US Centers for Disease Control and Prevention.

\section{Authors' contributions}

$\mathrm{BA}, \mathrm{RMO}$ and JEG wrote the protocol and designed the study. BA, TO, CW, AW, $\mathrm{BO}, \mathrm{DO}, \mathrm{MN}$, and $\mathrm{RMO}$, participated in data collection and sample analysis. $J E G, M J D, S M, E O, D W, K O$ and RMO, provided technical oversight during the study. BA, MM and RMO analyzed the data and prepared manuscript figures. $\mathrm{BA}$ and RMO. wrote the manuscript. All authors read and approved the final manuscript.

\section{Funding}

This study was supported by the United States President's Malaria Initiative through the United States Agency for International Development Africa Indoor Residual Spraying (AIRS) Project. The opinions expressed herein are those of the authors and do not necessarily reflect the views of the USAID, PMI or CDC

\section{Availability of data and materials}

All data generated or analyzed during this study are included in this published article and its additional files.

\section{Declarations}

\section{Ethics approval and consent to participate}

The study was approved by the Kenya Medical Research Institute/Scientific and Ethics Review Unit (KEMRI/SERU), number 2776, and by CDC through a reliance agreement with KEMRI/SERU (CDC IRB 6728). Individuals participating in outdoor trapping gave informed consent. Those conducting HLC were screened for malaria before the start of the study and treated if positive.

Collectors were placed on mefloquine malaria prophylaxis (Mephaquin, Acino Pharma AG, Switzerland) 1 week before collections began, with repeat doses once every week through the collection period, until 4 weeks after collections ended. Verbal consent was sought from the household head to use CDC-LT indoor and outdoor traps in their compound. All methods were performed in accordance with relevant guidelines and regulations.

\section{Consent for publication}

Not applicable.

\section{Competing interests}

The authors declare that they have no competing interests.

\section{Author details}

${ }^{1}$ Centre for Global Health Research, Kenya Medical Research Institute, P.O. Box 1578, Kisumu, Kenya. ${ }^{2}$ PMI VectorLink Project, Abt Associates Inc., Whitehouse, Milimani, Kisumu, Ojijo Oteko Road, P.O. Box 895-40123, Kisumu, Kenya. ${ }^{3}$ Division of Parasitic Diseases and Malaria, Center for Global Health, Centers for Disease Control and Prevention, Atlanta, GA 30333, USA. ${ }^{4}$ Liverpool School of Tropical Medicine, Pembroke Place, Liverpool L3 5QA, UK. ${ }^{5}$ PMI VectorLink Project, Abt Associates Inc., 6130 Executive Blvd, Rockville, MD 20852, USA. ${ }^{6}$ The United States President's Malaria Initiative (PMI), US Embassy Nairobi, United Nations Avenue, Nairobi, Kenya.

Received: 18 January 2021 Accepted: 20 May 2021

Published online: 12 June 2021

\section{References}

1. Dekker T, Takken W, Braks MA. Innate preference for host-odor blends modulates degree of anthropophagy of Anopheles gambiae sensu lato (Diptera: Culicidae). J Med Entomol. 2001;38(6):868-71.

2. WHO. World Malaria Report. Geneva: Word Health Organization; 2019.

3. Bhatt $\mathrm{S}$, et al. The effect of malaria control on Plasmodium falciparum in Africa between 2000 and 2015. Nature. 2015;526(7572):207-11.

4. Bayoh MN, et al. Anopheles gambiae: historical population decline associated with regional distribution of insecticide-treated bed nets in western Nyanza Province Kenya. Malar J. 2010;9:62.

5. McCann RS, et al. Reemergence of Anopheles funestus as a vector of Plasmodium falciparum in western Kenya after long-term implementation of insecticide-treated bed nets. Am J Trop Med Hyg. 2014;90(4):597-604.

6. Musiime AK, et al. Impact of vector control interventions on malaria transmission intensity, outdoor vector biting rates and Anopheles mosquito species composition in Tororo, Uganda. Malar J. 2019;18(1):445.

7. Abong'o $B$, et al. Impact of indoor residual spraying with pirimiphosmethyl (Actellic 300CS) on entomological indicators of transmission and malaria case burden in Migori County, western Kenya. Sci Rep. 2020;10(1):4518.

8. Ndenga BA, et al. Malaria vectors and their blood-meal sources in an area of high bed net ownership in the western Kenya highlands. Malar J. 2016:15:76.

9. Ototo EN, et al. Surveillance of malaria vector population density and biting behaviour in western Kenya. Malar J. 2015;14:244.

10. Killeen GF. Characterizing, controlling and eliminating residual malaria transmission. Malar J. 2014;13:330.

11. Degefa T, et al. Indoor and outdoor malaria vector surveillance in western Kenya: implications for better understanding of residual transmission. Malar J. 2017:16(1):443.

12. Sougoufara S, Ottih EC, Tripet F. The need for new vector control approaches targeting outdoor biting Anopheline malaria vector communities. Parasit Vectors. 2020;13(1):295.

13. Killeen GF, et al. Most outdoor malaria transmission by behaviourallyresistant Anopheles arabiensis is mediated by mosquitoes that have previously been inside houses. Malar J. 2016;15:225.

14. Vale GA, Torr SJ. Surveillance and sampling of disease vectors. Rev Sci Tech. 2015;34(1):227-33.

15. Service MW. Mosquito ecology: field sampling methods, 2nd ed. London: Elsevier; 1993. p 988.

16. Lindsay SW, et al. Variation in attractiveness of human subjects to malaria mosquitoes (Diptera: Culicidae) in The Gambia. J Med Entomol. 1993;30(2):368-73.

17. Mukabana WR, et al. Host-specific cues cause differential attractiveness of Kenyan men to the African malaria vector Anopheles gambiae. Malar J. 2002;1:17.

18. Ndebele P, Musesengwa R. View point: Ethical dilemmas in malaria vector research in Africa: making the difficult choice between mosquito, science and humans. Malawi Med J. 2012;24(3):65-8.

19. Achee $\mathrm{NL}$, et al. Considerations for the use of human participants in vector biology research: a tool for investigators and regulators. Vector-Borne Zoon Dis. 2015;15(2):2015.

20. Gimnig JE, et al. Incidence of malaria among mosquito collectors conducting human landing catches in western Kenya. Am J Trop Med Hyg. 2013;88(2):301-8. 
21. Onyango SA, et al. Monitoring malaria vector control interventions: effectiveness of five different adult mosquito sampling methods. J Med Entomol. 2013;50(5):1140-51.

22. Sikaala $\mathrm{CH}$, et al. Evaluation of alternative mosquito sampling methods for malaria vectors in Lowland South-East Zambia. Parasit Vectors. 2013;6:91.

23. Wong J, et al. Standardizing operational vector sampling techniques for measuring malaria transmission intensity: evaluation of six mosquito collection methods in western Kenya. Malar J. 2013;12:143.

24. Mathenge, E.M., et al., Comparative performance of the Mbita trap, CDC light trap and the human landing catch in the sampling of Anopheles arabiensis, An. funestus and culicine species in a rice irrigation in western Kenya. Malar J, 2005. 4: 7

25. Lines J, et al. Monitoring human-biting mosquitoes (Diptera: Culicidae) in Tanzania with light-traps hung beside mosquito nets. Bull Entomol Res. 1991:81(1):77-84.

26. Charlwood JD, et al. The Furvela tent-trap Mk 1.1 for the collection of outdoor biting mosquitoes. PeerJ. 2017;5: e3848.

27. Charlwood JD, et al. Feeding frequency and survival of Anopheles gambiae in a rice-growing area in Ghana. Med Vet Entomol. 2012;26:263-70.

28. Charlwood JD, et al. Effects of the spatial repellent metofluthrin on landing rates of outdoor biting anophelines in Cambodia Southeast Asia. Med Vet Entomol. 2016;30(2):229-34.

29. Hawkes FM, et al. Exploiting Anopheles responses to thermal, odour and visual stimuli to improve surveillance and control of malaria. Sci Rep. 2017;7(1):17283.

30. Vale GA. New field methods for studying the responses of tsetse flies (Diptera, Glossinidae) to hosts. Bull Entomol Res, 1974; 64:199-208.

31. Mboera LE, et al. Short report: influence of centers for disease control light trap position, relative to a human-baited bed net, on catches of Anopheles gambiae and Culex quinquefasciatus in Tanzania. Am J Trop Med Hyg. 1998;59(4):595-6.

32. Costantini $\mathrm{C}$, et al. Realationship to bitting colletions and influence of light and bednet in CDC light-trap catches of west Africa malaria vectors. Bull Entomol Res. 1998:88:503-11.

33. Mboera LE. Sampling techniques for adult Afrotropical malaria vectors and their reliability in the estimation of entomological inoculation rate. Tanzan Health Res Bull. 2005;7(3):117-24

34. Gillies MT, Coetzee M. A supplement to the anophelinae of Africa South of the Sahara (Sfrotropical region). 1987, Johannesburg: South African Institute for Medical Research.

35. MR4, Methods in Anopheles Research, 2015 ed.CDC Atlanta, Gorgia, USA. 2007.

36. Wirtz RA, et al. Comparative testing of monoclonal antibodies against Plasmodium falciparum sporozoites for ELISA development. Bull World Health Organ. 1987;65(1):39-45.
37. Scott JA, Brogdon WG, Collins FH. Identification of single specimens of the Anopheles gambiae complex by the polymerase chain reaction. Am J Trop Med Hyg. 1993;49(4):520-9.

38. Koekemoer $\mathrm{LL}$, et al. A cocktail polymerase chain reaction assay to identify members of the Anopheles funestus (Diptera: Culicidae) group. Am J Trop Med Hyg. 2002;66(6):804-11.

39. Fournier DA, et al. AD Model \{B\}uilder: using automatic differentiation for statistical inference on highly parameterized complex nonlinear models. Optimiz Methods Softw. 2012;27(2):233-49.

40. Abong'o B, et al. Host Decoy Trap (HDT) with cattle odour is highly effective for collection of exophagic malaria vectors. Parasit Vectors. 2018;11(1):533.

41. Maliti DV, et al. Development and evaluation of mosquito-electrocuting traps as alternatives to the human landing catch technique for sampling host-seeking malaria vectors. Malar J. 2015;14:502.

42. Govella NJ, et al. An improved mosquito electrocuting trap that safely reproduces epidemiologically relevant metrics of mosquito humanfeeding behaviours as determined by human landing catch. Malar J. 2016;15:465.

43. Sanou A, et al. Evaluation of mosquito electrocuting traps as a safe alternative to the human landing catch for measuring human exposure to malaria vectors in Burkina Faso. Malar J. 2019;18(1):386.

44. Meza FC, et al. Mosquito electrocuting traps for directly measuring biting rates and host-preferences of Anopheles arabiensis and Anopheles funestus outdoors. Malar J. 2019;18(1):83.

45. Bradley J, et al. Increased risks of malaria due to limited residual life of insecticide and outdoor biting versus protection by combined use of nets and indoor residual spraying on Bioko Island Equatorial Guinea. Malar J. 2012;11:242.

\section{Publisher's Note}

Springer Nature remains neutral with regard to jurisdictional claims in published maps and institutional affiliations.

Ready to submit your research? Choose BMC and benefit from

- fast, convenient online submission

- thorough peer review by experienced researchers in your field

- rapid publication on acceptance

- support for research data, including large and complex data types

- gold Open Access which fosters wider collaboration and increased citations

- maximum visibility for your research: over 100M website views per year

At BMC, research is always in progress.

Learn more biomedcentral.com/submissions 